\title{
Index
}

Ảbrams, M. H., 12n

Aesthetic ideology, viii- $x, 4-37,42,49-$ $55,57 \mathrm{n}, 63,68-69,134-35,142-43$, 171, 174, 201-2, 207-13. See also Aesthetics

Aesthetic judgment, 67-69; in Kant, 1220, 29-31, 103, 136

Aesthetic State, ix, 22-23, 50-51, 76-77, 96, 99n, 121-22, 124, 207-9, 212-13

Aesthetics: and Bildungsroman, viii, $\mathbf{x i}$, $42-43,52-56,63-65,134-35,202-3$, 209; and body, 33-34n, 89-93, 113-24, 126-33, 149, 155, 159-60, 203, 207; and bureaucracy, 28, 35-37, 94n, 209, 211; and canon, 26-28; critique of, 5, 33-34, 134, 170; and culture, 9-10, 12, 22-23, 25n, 27-29, 52-53, 142, 213; development of, 5-12; and exemplarity, 17-23, 49-51, 53-55, 68-69; and fascism, ix, 24-25, 98, 121-22, 207-8; and formalization, 13-23, 31, 42-43, 58, 96, 102-3, 208; and gender, 26n, 66, 203-7; and history, 10-12, 19-26, 48-51, 68-69, 141-44, 148, 171-72, 201-4, 213; and humanism, 12n, 39, 121-22; and ideology, viii-x, 4-5, 11, 33-34, 57n, 201-3; and irony, 6o-62, 65, 102-3, 148-49, 170, 172; and language, ix- $x, 29-35$; and literature, $x i, 28-29,44-46,53-54$, $62-64,79-80,124,202,213$; and na- tionalism, 22-24; and pedagogy, viii, xi-xii, 21, 26-28, 49-51, 68, 74-79, 94n, 146-50, 202, 210; and politics, viii-xii, 3-4, 7-10, 19-27, 50-51, 95-99, 11317, 121-22, 134-35, 149n, 201-13; and pragmatism, 17n, 105-19, 124, 210-13; and racism, ix, 24-25, 53, 97-98; and subject, viii, $6-7,26,46,50-53,61-62$, 67-69, 122, 143, 202; and technics, 9799, 104-9, 208; and theory, $x-x i, 4-5$, 27-37, 201-2, 210-13; and the university, ix, 27-28, 35-37, 45, 209-12.

Allen, Woody, $173 \mathrm{n}$

Althusser, Louis, 1, 34n

Ammerlahn, Hellmut, 7in

Amrine, Frederick, 41, 43

Anderson, Benedict, 23

Apter, Emily, 185

Arendt, Hannah, 122n, 209n

Aristotle, 97, 208

Armstrong, Bruce, 106n

Arnold, Matthew, 9, 12n, 22, 135

Ashton, Rosemary, 48n

Auerbach, Erich, 32

Auerbach, Nina, 153n

Author: and author-function, 102-4

Bahr, Ehrhard, 102, 103n

Baldick, Chris, 25n, 45n

Baldwin, Birgit, 104n, 111n 
Balibar, Étienne, 34n

Barnes, Julian, 173n

Barrell, John, 7n

Barthes, Roland, 31-32, 178

Baumgarten, Alexander, 6, 8

Beddow, Michael, 55, 56n, 73n, 79

Beer, Gillian, 161n

Bender, John, 139n

Benjamin, Walter, ix, 24, 44, 58-61, 184n, 199-200, 208, 208-9n

Bernal, Martin, 22n

Bernheimer, Charles, 18on

Bhabba, Homi, 23n, 24n

Bildung, 46-56, 59, 63-64, 96, 171-72, 201-3; in L'Education sentimentale, 179, 197, 200; in Middlemarch, 139, 142-44, 147-49; in Wilhelm Meisters Lehrjahre, 67-85; in Wilhelm Meisters Wanderjahre, 99, 105-24, 130, 133

Bildungsroman: vii-viii, xi-xii, 38-66, 134-35, 171-72, 202; and aesthetics, viii, xi, 42-43, 52-56, 63-65, 134-35, 202-3, 209; and L'Education sentimentale, 171-72, 200; as exemplary, 52-55, 65-66; and history, 53n, 56n, 171-72; and humanism, 39, 42-43, 55; and irony, 53-54, 58-62, 64-66, 171-72; and literary absolute, $x i, 39,52-53$; as phantom, xi, 41, 43, 57, 62, 63-64, 93, 134-35, 202; problems of definition of, $38-43,52-58,63-66,134-35,202-3$; and Wilhelm Meisters Lehrjahre, 65-66; and Wilhelm Meisters Wanderjahre, 100

Blackall, Eric, 88, 91n, 101n, 112, 119, 125, 13on, $133 \mathrm{n}$

Blanchot, Maurice, 44-45, 2oon

Bodmer, Johann Jakob, 8

Body, 33-34n, 136-38, 203, 207; in Eliot, 140-41, 149, 155, 159-60; in "Im ernsten Beinhaus" ("Schillers Reliquen"), 126-33; in Wilhelm Meisters Lehrjahre, 88-93; in Wilhelm Meisters Wanderjahre, 113-24. See also Aesthetics Borcherdt, Hans Heinrich, 53n

Borch-Jacobsen, Mikkel, 163, 163-64n, 165

Bourdieu, Pierre, 27n

Boyle, Nicholas, 119n

Breitinger, Johann Jakob, 8

Brombert, Victor, $178 \mathrm{n}, 190 \mathrm{n}$

Brooks, Peter, 175n, 185n, 19on
Brown, Jane K., 101n

Bruford, W. H., 48n, 99n, 101, 106n

Buckley, Jerome H., 41n

Bulwer-Lytton, Edward, 167, 170

Burckhardt, Jacob, 12n

Burd, Stephen J., $3 \mathrm{n}$

Bureaucracy: and aesthetics, 28, 35-37, 94n, 209, 211

Burke, Edmund, 7, 135, 136-37, 188, 207n

Butler, Judith, 206, 207

Butler, Samuel, 169-70

Cajuero-Roggero, Maria Amalia, 175n

Canon, 26-28

Carlyle, Thomas, 88n, 91n, 135n

Caruth, Cathy, 31n, 87, 197n, 204n

Casteux, P.-G., 188n

Catachresis, 31-32, 34, 87-88, 118, 168.

See also Language; Rhetoric

Caygill, Howard, 7-8, 9n, 10

Chase, Cynthia, 34, 87n, 204n, 209n

Chytry, Josef, 97n, 99n

Coleridge, Samuel Taylor, 10

Colvin, Sidney, 140

Commodity fetishism, 181-86. See also Fetish

Compagnon, Antoine, 45n

Cottom, Daniel, 144n

Court, Franklin E., 24n

Criticism, x, 43-46, 52, 54-66, 134. See also Literature; Theory

Culler, Jonathan, 82n, 172, 173n, 174, $178 \mathrm{n}, 190 \mathrm{n}, 193$

Culture, viii, 9-10, 12, 22-23, 25n, 27-29, 52-53, 142, 213; and multiculturalism, 26-27; and popular culture, 27-29, 5253. See also Aesthetics, Bildung

Czyba, L., 186n

Dällenbach, Lucien, 79n

David, Claude, 101n

Deconstruction, x, 2-4, 35-36, 201-2. See also Rhetorical Reading; Theory

Defoe, Daniel, 161n

Degoumois, Léon, 172n

de Graef, Ortwin, 155n

Deleuze, Gilles, 12, 68n

de Man, Paul, ix-x, 2-5, 31n, 32-37, 61n,

88, 116n, 155n, 201, 209n, 211 
Derrida, Jacques, 2-3, 16-17, 29, 68n, $80 n, 87 n, 103,112 n, 113 n, 117 n, 137$, 16o, 165, 182n, 197n, 200, 208

de Tracy, Destutt, 34

Dewey, John, 17n

Dilthey, Wilhelm, vii, 4on, 42

Dumesnil, René, 172n, 174n, 195n

During, Simon, 152-53n, 153, 154-55n

Eagleton, Terry, 4, 6, 136n, 138n, 144, 161n

Eckermann, Johann Peter, 100, 111, 125, 126n

Elias, Norbert, 12n, 48n

Eliot, George: Daniel Deronda, 139, 14445, 154, 156-60, 162, 165-66; Impressions of Theophrastus Such, 167-70; The Lifted Veil, 160-62; Middlemarch, xi, 139-56, 160, 166, 171, 203; "The Natural History of German Life," 139, 14243; "Women in France," 166

Eliot, T. S., 10

Engels, Friedrich, 34, 174n, 182n, 20on, 213

Entsagung. See Renunciation

Exemplarity, 17-23, 49-51, 53-55, 68-69. See also Aesthetics, Bildung

Fanon, Franz, 199

Fascism, ix, 4, 24-25, 49n, 53, 98, 122, 135, 207-8

Fatherhood: in Wilhelm Meisters Lehrjahre, 70-71, 73, 81-85, 89-90. See also Gender; Phallocentrism

Fehér, Ferenc, 55-56

Feise, Ernst, 133n

Felman, Shoshana, 122n

Fenves, Peter, 22n

Ferguson, Frances, 15n

Ferris, David, 153n

Fetish, 2, 18o-84, 206n, 209; in L'Education sentimentale, 184-200. See also Commodity fetishism

Fish, Stanley, 210

Flaubert, Gustave: L'Education sentimentale, xi-xii, 171-200, 203, 207; Madame Bovary, 152, 172-73, 179-80, 185n

Foucault, Michel, 11-12, 16-17, 44n, 5152n, 94n, 102-3n, 173

Freud, Sigmund, 71, 82, 87, 135, 138, 163$66,183-84,196 \mathrm{n}$
Fuderer, Laura Sue, 66n

Furet, François, 199

Gadamer, Hans-Georg, 10, 46-50, 54, 68

Gaillard, Françoise, 188n

Gender, 73, 93, 84n, 120n; and aesthetics, 26n, 66, 203-7. See also Fatherhood; Maternal; Phallocentrism

Gide, André, 79n

Gilbert, Sandra, 153n, 153-54, 161n

Gille, Klaus F., 41n

Ginsburg, Michal Peled, 185n

Gobineau, Arthur, comte de, 24-25

Godzich, Wlad, 211-13

Goebbels, Joseph, 126-27n

Goethe, Johann Wolfgang von: "Im ernsten Beinhaus" ("Schillers Reliquen"), 125-33; Wilhelm Meisters Lehrjahre, xi, 38, 40-41, 59-61, 65-94, 1057, 109, 120, 130, 138, 171-72, 203-4, 207, 209; Wilhelm Meisters Wanderjahre, xi-xii, 92n, 99-124, 125-27, 171, 203, 207-9

Gottsched, Johann Christoph, 8

Graff, Gerald, 45n

Grimm, Hans, 53n

Gubar, Susan, 153n, 153-54, 161n

Guillory, John, 2-3, 9n, 28-29, 35-36, 211-13

Haig, Stirling, 172n

Haight, Gordon, 168, 170

Halperin, David, 205n

Hazlitt, William, 137n

Heath, Stephen, 206n

Hecker, Max, 126n

Hegel, G. W. F., 6, 11n, 21, 32n, 39, 46, $48-49,53,60-62,65,88 \mathrm{n}, 134,143$, $204 \mathrm{n}$

Heidegger, Martin, 92n, 96-98, 111-12n, 122-24, 167n, 208

Herder, Johann Gottfried, 8, 10, 46

Herf, Jeffrey, 98n, 209n

Herschberg-Pierrot, Anne, 175n, 188n

Hertz, Neil, 120n, 144n, 145-47, 156-59, $162,167,188-89$ n, 204n

Hirsch, Marianne, $38 \mathrm{n}$

History, 171, 197-200; and aesthetics, 10$12,19-23,25-26,48-51,68-69,171-$ 72, 197-200, 201-4, 213; in Eliot, 141- 


\section{History (continued)} 44, 148, 167-68; in L'Education sentimentale, 174-80, 186-200

Hohendahl, Peter, 45n

Hörisch, Jochen, 53n, 72n, 8on

Humanism. See Aesthetics; Bildung; Culture

Humbolt, Wilhelm von, 127n

Hume, David, 18-19, 135

Hutcheson, Francis, 7

Ideology, viii-x, 4-5, 11, 33-35, 201-2. See also Aesthetic ideology; Aesthetics Irigaray, Luce, 205n

Irony, 53-54, 58-62, 64-66; in Eliot, $148-$ 50, 170; in Flaubert, 172, 176-78, 191, 193; in Schlegel, 59-62, 74; in Wilhelm Meisters Lehrjahre, 74-81, 93; in Wilhelm Meisters Wanderjahre, 102-3

Jameson, Fredric, 4, 173-74, 181, 197 Jameson, Maureen, $185 \mathrm{n}$ JanMohammed, Abdul, 95n Jean, Raymond, $173 \mathrm{n}$ Johnson, Barbara, 2n, 204-5n Jost, François, 57n Joyce, James, 82n Jünger, Ernst, 208-9n

Kafka, Franz; 209

Kames, Henry Home, Lord, 7

Kamuf, Peggy, 210n

Kant, Immanuel, 5-6, 8n, 11n, 12-20, 22, 25-26, 29-31, 33n, 68, 96, 103, 136

Keenan, Thomas, $182 \mathrm{n}$

Keller, Gottfried, 41n

Kittler, Friedrich, 51n, 94n, 103n, 203n, 209

Kleist, Heinrich von, 21n, 88n

Klopstock, Friedrich Gottlob, 135, 138

Knapp, Steven, 21on

Kontje, Todd, 42n, 54n, 64

Kowalik, Jill Anne, 73n

Kristeva, Julia, 87n, 204n

Kruger, Loren, 23-24n

Lacan, Jacques, 81, 87, 165, 206

Lacoue-Labarthe, Philippe, 23, 25, 28n, $35,39-40,43-46,48-49,52-53,57 n$, $58,61,76,97-98,122,143,205-6$
Language, ix, 29-35, 48, 58-62, 73, 87-88, 93, 115-18, 123-24, 132, 136, 166-68, $171,187,207-8$. See also Rhetoric

Lanzmann, Claude, $122 \mathrm{n}$

Laplanche, Jean, 87n, 195-96

Leavis, F. R., 145

Le Bon, Gustave, 163-64

Lehman, David, $3 n$

Lentricchia, Frank, $3 n$

Levine, George, 140

Lewes, George Henry, 140, 143

Literary absolute, $x, 39,43-53,55,58,61$, 202; and Wilhelm Meisters Lehrjahre, 76, 79-80. See also Aesthetics; Literature; Subject

Literary theory. See Theory

Literature, viii, $x-x i, 28-29,37,43-46,49$, 53-54, 57-58, 62-64, 94n, 124, 202, 213; and criticism, x, 43-46, 52, 54; institution of, viii, 35-37, 43-45, 51n, 209-13; in L'Education sentimentale, 172-74, 179-80, 198-200; in Middlemarch, 15156; and Wilhelm Meisters Lehrjahre, 7980

Lloyd, David, 19, 22n, 25, 51, 56, 68n, 95n Locke, John, 204n

Ludendorff, Mathilde von, 126-27n

Lukács, Georg, 64, 75, 77, 79, 173

Lyotard, J.-F., 27n

MacLeod, Catriona, 92n

Mallarmé, Stéphane, 172

Mann, Thomas, 99

Marshall, David, 136

Martini, Fritz, 4on

Marx, Karl, 34, 174, 181-83, 198, 20on, 213

Materiality, 33-34, 88, 132-33, 162, 196

Maternal: in Daniel Deronda, 156-57; in L'Education sentimentale, 187, 204; in Wilhelm Meisters Lehrjahre, 72-73, 8587, 92-93, 204. See also Gender

Mautner, Franz H., 131n, 133n

May, Kurt, 4in

Mehlman, Jeffrey, 87n

Michaels, Walter Benn, 210n

Michaud, Éric, 25n

Miller, J. Hillis, 91n, 141, 143

Moretti, Franco, 56, 57

Morgenstern, Karl, 4on, 54

Mosse, George L., 24n 
Muenzer, Clark, 107, 108

Multiculturalism, 3, 26-27

Musil, Robert, 42-43, 52

Nancy, Jean-Luc, 25, 28n, 39-40, 43-46,

$49,52-53,57 \mathrm{n}, 58,61,76,98,143$

Nationalism, 22-24

Neuhaus, Volker, 101n, 103

Newman, John Henry, Cardinal, 9-10

Newmark, Kevin, 61

Nietzsche, Friedrich, 143n

Novalis (Friedrich von Hardenberg), 41n

Ohmann, Richard, 1on

Orr, Mary, 187n

Pabst, Walter, 41n

Pedagogy: and aesthetics, viii, xi-xii, 21, 26-28, 49-51, 68, 74-79, 94n, 146-50, 202, 210. See also Bildung

Perruchot, Claude, 178n

Peschken, Bernd, 113, 115n

Phallocentrism, 66n, 186, 204. See also Gender

Pietz, William, 181n

Plato, 5, 97, 205

Pocock, J. G. A., 8n

Politics: and aesthetics, viii-xii, 3-5, 7$10,19-27,50-51,95-99,113-17,121-$ 22, 134-35, 149n, 201-13

Pontalis, J.-B., 195-96

Poovey, Mary, 26n, 206n

Pragmatism: and aesthetics, 17n, 105-19, 124, 210-13

Prendergast, Christopher, $178 \mathrm{n}$

Prosopopeia, 34, 88. See also Language; Rhetoric

Proust, Marcel, 179

Raabe, Wilhelm, 41n

Racism: and aesthetics, ix, 24-25, 53, 9798

Readings, Bill, 27n, 211n

Redfield, Marc, 3n, 27n, 34n, 59n

Reed, Arden, 16n

Renunciation (Entsagung), 74-81, 106-9, 111-12, 116

Rhetoric, 29-34, 58-62, 64-66, 71-73, 8793, 115-19, 121-24, 130-33, 136-38, 145-46, 149-50, 155, 166-68, 170, 171,
174, 176-80, 190-93, 202-9. See also

Catachresis; Irony; Language;

Prosopopeia

Rhetorical reading, $x, 32-37,130-33,211$

Riffaterre, Michael, 179

Ronell, Avital, $165 \mathrm{n}$

Rose, Jacqueline, 147n, 153n, 159-6o, 206n

Rose, Mark, 44n

Rosenberg, Alfred, 24, 25n

Roustang, François, 165n

Royle, Nicholas, 16o, 162, 165-66

Ruppelt, Georg, 127n

Sagave, Pierre-Paul, 99n

Sammons, Jeffrey, 38, 41, 43, 53n, 57

Sartre, Jean-Paul, 172-73, 180, 187, 204

Savage, Eliza, 169-70

Scarry, Elaine, $138 \mathrm{n}$

Schaffner, Randolph P., 38n

Schelling, F. W. J. von, 11n

Schiller, Friedrich, viii-ix, xi, 20-22, 25, $32,49-52,66-75,80-81,84 \mathrm{n}, 85,88$, 93, 96, 125-33, 134, 204, 213; and Wilhelm Meisters Lehrjahre, 66-74

Schlegel, Friedrich, 39, 45, 59-62, 65-66, 74, 80, 93, 134

Schrader, Monika, 52, 54n

Schwabe, Carl Lebrecht, 126-27

Sedgwick, Eve Kosofsky, 138, 169n

Seltzer, Mark, 138n

Sentiment, 135-38, 141-44, 146. See also Sympathy

Sentimentalism. See Sentiment

Shaftesbury, Anthony Ashley Cooper,

Third Earl of, xi, 7, 135, 206n

Shuttleworth, Sally, 140n, 151n

Simpson, David, 36n

Smith, Adam, 8-11, 15

Spivak, Gayatri Chakravorty, 182n

Sprinker, Michael, in, 34n

Stiegler, Bernard, 209n

Stifter, Adalbert, 4in

Stolnitz, Jerome, $7 \mathrm{n}$

Subject: and aesthetics, viii, 6-7, 26, 46, $50-53,61-62,67-69$, 94n, 122, 143, 180-82, 202. See also Bildung; Literary absolute

Sublime, 15, 167-68

Swales, Martin, 55 
Swann, Charles, 161n

Symbol: in Critique of Judgment, 30-32; in L'Education sentimentale, 175-76, 190; in Middlemarch, 147-48; in Wilhelm Meisters Wander jahre, 101-5, 108, 110-12, 116, 118-19, 124, 209. See also Aesthetics and pragmatism

Sympathy: and aesthetics, 135-38; in Eliot, 138-39, 142-49, 156, 159, 161-63, 169-70. See also Sentiment

Tanner, Tony, 18 on

Tarde, Gabriel, 163-64

Taylor, Françoise M., 24n

Technics, 104-9, 122-24, 165-68, 208; as techne, 97-99, 105, 122-24, 208; and aesthetics, 96-97, 105-24, 208; and question of technology, 98n, 111n, 12224,208

Technology. See Technics

Telepathy, 160-70

Theory, $x-x i, 1-5,44-46,61,63-64$; and aesthetics, viii, 4-5, 27-37, 201-2, 21013. See also Criticism; Literature

Thibaudet, Albert, 179
Trousseau, A., 195

Tunner, Erika, 71-72n, 92n

University: and aesthetics, ix, 27-28, 35$37,45,209-12$

Veil, Wolfgang, 126n

Viëtor, Karl, 126n, 127n, 129, 130n, 131n, 132n

Viswanathan, Gauri, 25n

von Mücke, Dorothea, 52n

Wagner, Richard, 24n

Warner, Michael, 45n

Weber, Samuel, 58-59, 123

Wieland, Christoph Martin, 41n

Williams, Raymond, 7n, 12n, 48n

Winckelmann, Johann Joachim, 10n, 23, 207n

Woodmansee, Martha, 28n, 44n, 95-96n

Wundt, Max, 126n

Young, Robert, 1on

Žižek, Slavoj, 196

Zola, Emile, 151n, 173n 